\title{
Dreibein as Prepotential for Three-Dimensional Yang-Mills Theory
}

\author{
Indrajit Mitra ${ }^{1}$ and H. S. Sharatchandra ${ }^{2}$ \\ ${ }^{1}$ Department of Physics, University of Calcutta, 92 APC Road, Kolkata 700009, India \\ ${ }^{2}$ Centre for Promotion of Research, 7 Shaktinagar Main Road, Porur, Chennai 600116, India \\ Correspondence should be addressed to Indrajit Mitra; indra.theory@gmail.com
}

Received 6 April 2017; Revised 15 June 2017; Accepted 9 July 2017; Published 22 August 2017

Academic Editor: Edward Sarkisyan-Grinbaum

Copyright (c) 2017 Indrajit Mitra and H. S. Sharatchandra. This is an open access article distributed under the Creative Commons Attribution License, which permits unrestricted use, distribution, and reproduction in any medium, provided the original work is properly cited. The publication of this article was funded by SCOAP ${ }^{3}$.

\begin{abstract}
We advocate and develop the use of the dreibein (and the metric) as prepotential for three-dimensional SO(3) Yang-Mills theory. Since the dreibein transforms homogeneously under gauge transformation, the metric is gauge invariant. For a generic gauge potential, there is a unique dreibein on fixing the boundary condition. Topologically nontrivial monopole configurations are given by conformally flat metrics, with scalar fields capturing the monopole centres. Our approach also provides an ansatz for the gauge potential covering the topological aspects.
\end{abstract}

\section{Introduction}

In this paper we advocate and develop the use of the dreibein or triad (the 3D version of the vielbein) as the basic variable or prepotential for Yang-Mills theory. The first clear proposal to use the dreibein came from Haagensen and Johnson [1] in the context of Hamiltonian formalism in $3+1$ dimensions. (References [2-8] are some other works on Yang-Mills theory involving various constructions of the metric.) The defining equation for the dreibein is the condition for the dreibein to be torsion-free with respect to a connection one-form. The authors of [1] claimed that there are zero modes associated with this equation due to nonuniqueness of the dreibein $e_{a}^{i}$ corresponding to a given gauge potential $A_{i}^{a}$ but also gave arguments that the zero modes would not affect their discussion. Then Haagensen et al. [9] followed it up with a deformation of the defining equation for the dreibein, removing the deformation at the end. References [5-8] have used the original defining equation for the dreibein as in [1] and not the deformation of it. In this work also, we use the original defining equation of [1]. We analyse the case of a generic Yang-Mills potential, satisfying $\operatorname{det} B_{i}^{a} \neq 0\left(B_{i}^{a}\right.$ being the nonabelian magnetic field) and argue that a unique dreibein is obtained by fixing the boundary condition. Consequently, the torsion-free dreibein is a useful prepotential. The calculation of the Jacobian will be affected by the zero modes present for vanishing nonabelian magnetic field, but the Jacobian will not be needed in the present work.

Our formulation is useful in several ways:

(1) The metric arising out of the dreibein is gauge invariant, and so the topological properties of the field configurations which we link to it are also gauge invariant. The Yang-Mills action is like $R^{2}$ theory, but without the diffeomorphism invariance. It should be noted that our interest in the present work is about Yang-Mills theory, not gravity.

(2) Our formulation can be useful for a nonperturbative understanding of Yang-Mills theory. We find that the topological, monopole configurations (which may drive confinement) correspond to conformally flat metrics. In our earlier works $[5,10]$, we showed how to locate such a configuration to an internal point (the "centre") in a gauge invariant way. In this work, the "centres" are identified as certain points at which the conformal mode (a scalar field) is extremum.

(3) There has been extensive interest in obtaining an ansatz for the Yang-Mills potential that exhibits the topological aspects [11-16]. We propose an ansatz 
which has a scalar and a spin-two part in addition to a pure gauge. The scalar part comes from the conformally flat metric which captures the monopole configurations.

Our techniques can be extended in a straightforward way to SO(4) Yang-Mills theory in 4-Euclidean dimensions. Haagensen and Johnson [1] have also addressed generalization to other gauge groups. Our approach can also be applied to the $3+1$-dimensional Yang-Mills theory in the Hamiltonian formulation, with the physical states described as wave functionals of the metric.

Ever since the proposal of nonabelian gauge theory, the similarity with Einstein gravity has led to extensive work exploring the relationship between the two. Examples of this on the gravity side include the Chern-Simons formulation of $2+1$ gravity [17], Ashtekar and loop gravity formulation [18], and the pure connection formulation of general relativity [19]. We have already cited several works which attack gauge theory using gravity [1-9]; a recent work is [20]. In spite of all this, our approach gives a new way to attack the exotic features of Yang-Mills theory.

The paper is arranged as follows. In Section 2, the dreibein $e_{i}^{a}$ is defined through the torsion-free condition, and the gauge invariance of the metric is emphasized. In Section 3, we analyse the existence and uniqueness of $e_{i}^{a}$ for a given gauge potential $A_{i}^{a}$. Section 4 deals with topological (monopole) field configurations and Section 5 presents the gauge potential ansatz. In Section 6, we discuss our results.

\section{Dreibein as Prepotential}

Consider the set of nine first-order partial differential equations (see, e.g., [1])

$$
\epsilon_{i j k}\left(\partial_{j} e_{k}^{a}+A_{j}^{a b} e_{k}^{b}\right)=0
$$

which constitute the "torsion-free condition" for the dreibein $e_{i}^{a}(x)(i=1,2,3$ are the space indices and $a=1,2,3$ are the group indices) with respect to a connection one-form $A_{i}^{a b}(x)$. The dreibein $e_{i}^{a}$ is viewed as the square root of a metric

$$
g_{i j}(x)=e_{i}^{a}(x) e_{j}^{a}(x) .
$$

If $\operatorname{det} e_{i}^{a}(x) \neq 0$, we can expand the LHS of (1) in the $e_{i}^{a}$ basis and write

$$
\partial_{j} e_{k}^{a}+A_{j}^{a b} e_{k}^{b}=\Gamma_{j k}^{l} e_{l}^{a}
$$

Imposing the symmetry

$$
\Gamma_{j k}^{l}=\Gamma_{k j}^{l}
$$

is equivalent to the torsion-free condition (1). Define

$$
\mathscr{D}_{i} e_{j}^{a}=\partial_{i} e_{j}^{a}+A_{i}^{a b} e_{j}^{b}-\Gamma_{i j}^{l} e_{l}^{a} .
$$

Then (3) is $\mathscr{D}_{j} e_{k}^{a}=0$, and so $\left[\mathscr{D}_{i}, \mathscr{D}_{j}\right] e_{k}^{a}=0$. This gives

$$
R_{k i j}^{l} e_{l}^{a}-F_{i j}^{a b} e_{k}^{b}=0
$$

where $R_{k i j}^{l}$ is the Riemann tensor and

$$
F_{i j}^{a b}=\partial_{i} A_{j}^{a b}-\partial_{j} A_{i}^{a b}+\left[A_{i}, A_{j}\right]^{a b}
$$

(see, e.g., [21]). Using the inverse matrix $\left\{e^{b k}\right\}$,

$$
e_{k}^{a} e^{b k}=\delta^{a b}
$$

we can write

$$
F_{i j}^{a b}=R_{k i j}^{l} e_{l}^{a} e^{b k}
$$

It may be helpful to link the above with Cartan's structure equations of general relativity. Cartan's first equation,

$$
d e^{a}+\omega^{a}{ }_{b} \wedge e^{b}=0
$$

is our equation (1); we have used the symbol $A$ in the place of $\omega$ and and put all group indices as upper indices. (Since we have $\mathrm{SO}$ (3) and not the Lorentz group, there is no need to distinguish between upper and lower indices.) Cartan's other equation

$$
R_{b}^{a}=d \omega_{b}^{a}+\omega_{c}^{a} \wedge \omega_{b}^{c}
$$

in components is (on using the dreibein to convert the group indices of $R^{a}{ }_{b i j}$ to space indices)

$$
R_{k i j}^{l} e_{l}^{a} e^{b k}=\partial_{i} \omega_{j}^{a}{ }_{b}-\partial_{j} \omega_{i b}^{a}+\left[\omega_{i}, \omega_{j}\right]_{b}^{a} .
$$

Equation (12) is (7) and (9) combined (in our notation).

Equation (1) can be written as

$$
\epsilon_{i j k}\left(D_{j} e_{k}\right)^{a}=0
$$

where $D_{j}$ is the gauge-covariant derivative:

$$
D_{j}^{a b}=\delta^{a b} \partial_{j}+A_{j}^{a b} .
$$

Now write

$$
A_{i}^{a b}=-\epsilon^{a b c} A_{i}^{c},
$$

where $A_{i}^{c}$ is the Yang-Mills potential. Then (7) gives the YangMills field strength. $A_{i}^{a}$ transforms inhomogeneously under an $\mathrm{SO}(3)$ gauge transformation, but (13) ensures that the dreibein $e_{i}^{a}$ transforms homogeneously:

$$
e_{i}^{a \prime}(x)=O^{a b}(x) e_{i}^{b}(x) .
$$

Here $O^{a b}$ is an $\mathrm{SO}(3)$ matrix. The metric $g_{i j}(x)$ as given by (2) is therefore gauge-invariant. All gauge invariant objects can be rewritten in terms of $g_{i j}$. For instance,

$$
\operatorname{tr}\left(F_{i_{1} j_{1}} F_{i_{2} j_{2}} \cdots\right)=\operatorname{tr}\left(R_{\left(i_{1} j_{1}\right)} g^{-1} R_{\left(i_{2} j_{2}\right)} g^{-1} \cdots\right),
$$

where $R_{(i j)}$ is the matrix with the elements $\left(R_{(i j)}\right)_{k l}=R_{k l i j}$ and $g^{-1}$ has the elements $\left(g^{-1}\right)_{k m}=g^{k m}$. This may be obtained from $F_{i j}^{a b}=R_{k l i j} g^{k m} e_{m}^{a} e^{b l}$. 
In 3 space dimensions, the Riemann curvature tensor can be completely expressed in terms of the Einstein tensor:

$$
R_{k l i j}=-g \epsilon_{k l m} \epsilon_{i j n} G^{m n}
$$

where $g=\operatorname{det} g_{i j}$. This allows us to express the Yang-Mills action in $3 \mathrm{D}$

$$
S=\frac{1}{2 g_{Y M}^{2}} \int d^{3} \times B_{i}^{a} B_{i}^{a},
$$

(where $B_{i}^{a} \equiv(1 / 2) \epsilon_{i j k} F_{j k}^{a}$ ) also in terms of the Einstein tensor, as follows. In (6), we use (18) and also $F_{i j}^{a b}=-\epsilon^{a b c} F_{i j}^{c}$. This gives [1]

$$
B_{i}^{a}=(\operatorname{det} e) e^{a j} G_{i j}
$$

where $\operatorname{det} e=\operatorname{det} e_{i}^{a}=\sqrt{g}$. Then the Yang-Mills action in 3D is

$$
S=\frac{1}{2 g_{\mathrm{Y} M}^{2}} \int d^{3} x g g^{j k} G_{i j} G_{i k} .
$$

This is like $R^{2}$ gravity, but without the diffeomorphism invariance.

Equation (1) or (13) comprises a set of linear equations for the variables $A_{i}^{a}$ :

$$
\epsilon_{i j k}\left(\partial_{j} e_{k}^{a}+\epsilon^{a b c} A_{j}^{b} e_{k}^{c}\right)=0 .
$$

Equation (22) has diffeomorphism covariance provided both $A_{i}^{a}$ and $e_{i}^{a}$ transform as covariant GL(3) vectors (the Christoffel symbol does not contribute due to antisymmetry of $\epsilon_{i j k}$ ). Note that $\epsilon_{i j k}$ in (22) can be replaced by the Levi-Civita tensor $\varepsilon^{i j k}$ since the two differ by just a factor of $\sqrt{g}$.

When the matrix $\left\{e_{i}^{a}\right\}$ is nonsingular, (22) can be uniquely solved for $A_{i}^{a}$. Indeed, multiplying by $e_{l}^{a}$ and summing over $a$ we get

$$
\epsilon_{i j k} e_{l}^{a} \partial_{j} e_{k}^{a}=a_{i l}-\delta_{i l} a_{m m}
$$

where $a_{j m}=(\operatorname{det} e) A_{j}^{b} e^{b m}$. Now set $i=l$ in (23) to get $a_{m m}$ and put it back in (23). Therefore $[1,5]$

$$
A_{l}^{b}=\frac{1}{\operatorname{det} e} \epsilon_{i j k} e_{l}^{a}\left(e_{i}^{b} \partial_{j} e_{k}^{a}-\frac{1}{2} \delta_{a b} e_{i}^{c} \partial_{j} e_{k}^{c}\right) .
$$

To sum up, (1) or (13) or (22) defines $e_{i}^{a}$. In our context, the dreibein does not arise from transformation between coordinate basis and orthonormal basis. The gauge transformation of $A_{i}^{a}$ ensures that $e_{i}^{a}$ transforms homogeneously and so $g_{i j}$ as defined by (2) is gauge invariant. Equation (22) is diffeomorphism covariant. But the action is not diffeomorphism invariant, since we are dealing with YangMills theory in flat space and not in curved space.

\section{On Existence and Uniqueness of $e_{i}^{a}$ for Given $A_{i}^{a}$}

When $A_{i}^{a}(x)=0,(22)$ implies $e_{i}^{a}$ is curl-free for each $a=$ $1,2,3$ and we have a general solution

$$
e_{i}^{a}(x)=\partial_{i} \varphi^{a}(x),
$$

where $\varphi^{a}(x)$ are arbitrary functions. Thus (22) has a large set of zero modes. In this situation, the curvature $F_{i j}^{a b}$ (or $R_{k l i j}$ ) vanishes and we have a flat space. From (2), we see that (25) corresponds to $g_{i j}(x)=\partial_{i} \varphi^{a} \partial_{j} \varphi^{a}$, that is, a set of curvilinear coordinates $\phi^{a}(x)$ of the flat space. Now (22) is covariant under diffeomorphisms. If we start with $A_{i}^{a}(x)=$ 0 and equation (25) and apply the diffeomorphism $x \rightarrow$ $x^{\prime}(x)$ using the transformation property of covariant vectors, $A_{i}^{a}$ stays zero but $e_{i}^{a}$ changes to $e_{i}^{a \prime}(x)=\partial_{i} \theta^{a}(x)$ (where $\left.\theta^{a}\left(x^{\prime}\right)=\phi^{a}(x)\right)$; that is, we have a new dreibein for the same $A_{i}^{a}$. Thus we may view the set of zero modes of (22) given by (25) as accidental to the case of $A_{i}^{a}=0$ and as a result of diffeomorphism covariance.

In [1], it is claimed that all pure gauges, and not just $A_{i}^{a}=0$, give zero modes. But a pure gauge potential $A_{i}(x)=$ $O^{T}(x) \partial_{i} O(x)$ (where $A_{i}^{a b}=-\epsilon^{a b c} A_{i}^{c}$ and $O$ is an $\operatorname{SO}(3)$ matrix) changes under both diffeomorphism and gauge transformation. If $A_{i}^{a}$ were invariant but the dreibein changed under a transformation, we would have had nonunique $e_{i}^{a}$ for a given pure gauge $A_{i}^{a}$. This does not appear to be the case. So, while we do not exclude zero modes for nonvanishing pure gauges, such zero modes do not follow from any symmetry (either gauge covariance or diffeomorphism covariance) of (22), unlike the zero modes for vanishing gauge potential.

Now both vanishing gauge potential and pure gauge $A_{i}^{a}$ correspond to $B_{i}^{a}=0$. The point we make in this section is that, for a generic gauge potential with $\operatorname{det} B_{i}^{a} \neq 0$, we do not have such large set of nonunique $e_{i}^{a}$. This issue was earlier addressed in Section 2 of [22]. Here we present a simpler and neater analysis, arriving at the same conclusion.

First we note that operating on (13) by $D_{i}$ gives a consistency condition, as follows. We have $\epsilon_{i j k} D_{i}^{c a} D_{j}^{a b} e_{k}^{b}=0$. Using antisymmetry of $\epsilon_{i j k}$ and $\left[D_{i}, D_{j}\right]^{c b}=F_{i j}^{c b}=-\epsilon^{c b a} F_{i j}^{a}$, we arrive at a condition to be satisfied by any solution to (22):

$$
\vec{B}_{i}(x) \times \vec{e}_{i}(x)=0 .
$$

In the case $\operatorname{det} B_{i}^{a} \neq 0$, without any loss of generality, we may expand $\vec{e}_{i}(x)$ in the basis provided by $\vec{B}_{i}(i=1,2,3)$ :

$$
e_{i}^{a}(x)=B_{j}^{a} \alpha_{j i}
$$

Putting (27) in the consistency condition (26) gives us

$$
(\operatorname{det} B) \epsilon_{i j k}\left(B^{-1}\right)_{k}^{a} \alpha_{j i}=0 .
$$

Multiplying (28) by $B_{l}^{a}$, we get $\epsilon_{i j l} \alpha_{j i}=0$. Therefore $\alpha_{i j}(x)$ is a symmetric matrix:

$$
\alpha_{i j}(x)=\alpha_{j i}(x)
$$

In terms of these variables $\alpha_{i j}$, (22) becomes

$$
\epsilon_{i j k}\left(\partial_{j} \alpha_{m k}+\beta_{m j l} \alpha_{l k}\right)=0
$$

with $i, j, k, l, m=1,2,3$. Here

$$
\beta_{m j l}=\left(B^{-1}\right)_{m}^{a}\left(D_{j} B_{l}\right)^{a} .
$$


Equation (30) gives the following six equations for evolution of $\alpha_{i j}$ in $x_{3}$ :

$$
\begin{aligned}
& \partial_{3} \alpha_{11}-\partial_{1} \alpha_{13}+\beta_{13 l} \alpha_{l 1}-\beta_{11 l} \alpha_{l 3}=0, \\
& \partial_{3} \alpha_{21}-\partial_{1} \alpha_{23}+\beta_{23 l} \alpha_{l 1}-\beta_{21 l} \alpha_{l 3}=0, \\
& \partial_{3} \alpha_{31}-\partial_{1} \alpha_{33}+\beta_{33 l} \alpha_{l 1}-\beta_{31 l} \alpha_{l 3}=0, \\
& \partial_{3} \alpha_{12}-\partial_{2} \alpha_{13}+\beta_{13 l} \alpha_{l 2}-\beta_{12 l} \alpha_{l 3}=0, \\
& \partial_{3} \alpha_{22}-\partial_{2} \alpha_{23}+\beta_{23 l} \alpha_{l 2}-\beta_{22 l} \alpha_{l 3}=0, \\
& \partial_{3} \alpha_{32}-\partial_{2} \alpha_{33}+\beta_{33 l} \alpha_{l 2}-\beta_{32 l} \alpha_{l 3}=0 .
\end{aligned}
$$

Note that there is no evolution equation for the variable $\alpha_{33}$. Also, since there are two evolution equations (33) and (35) for the same variable $\alpha_{12}=\alpha_{21}$, we get the consistency requirement

$$
\begin{aligned}
\partial_{3} \alpha_{12} & =\partial_{2} \alpha_{13}+\beta_{12 l} \alpha_{l 3}-\beta_{13 l} \alpha_{l 2} \\
& =\partial_{1} \alpha_{23}+\beta_{21 l} \alpha_{l 3}-\beta_{23 l} \alpha_{l 1} .
\end{aligned}
$$

This has to be satisfied at all $x_{3}$. The variable $\alpha_{33}$ enters in this consistency equation. The coefficient of $\alpha_{33}$ is $\beta_{123}-\beta_{213}$. If

$$
\beta_{123}-\beta_{213} \neq 0
$$

we can solve for $\alpha_{33}$ in favour of the other five variables $\alpha_{11}, \alpha_{12}, \alpha_{13}, \alpha_{22}, \alpha_{23}$. Substituting this for $\alpha_{33}$ in the (five) independent equations (32)-(37), we get evolution equations for these five independent variables. The solution is unique with initial choice on $x_{3}=x_{3}^{0}$ surface. This is ensured by the Cauchy-Kowalevski theorem (see, e.g., [22], which contains further reference, for a statement of this theorem). In the generic case, at least one of $\left(\beta_{123}-\beta_{213}\right),\left(\beta_{231}-\beta_{321}\right)$, and $\left(\beta_{312}-\beta_{132}\right)$ will be nonzero. If $\beta_{231}-\beta_{321} \neq 0$ (or $\beta_{312}-$ $\beta_{132} \neq 0$ ), we can consider the evolution equation in $x_{1}$ (or $x_{2}$ ) instead of $x_{3}$ and obtain unique solution with initial choice on $x_{1}=x_{1}^{0}\left(\right.$ or $\left.x_{2}=x_{2}^{0}\right)$ surface.

We have demonstrated that, on fixing the boundary condition, a unique torsion-free dreibein $e_{i}^{a}$ exists for a generic Yang-Mills potential $A_{i}^{a}$. So, after taking into account the zero modes discussed in the first two paragraphs of this section, it should be possible to calculate the Jacobian of the transformation. Thus the transformation from $A_{i}^{a}$ to $e_{i}^{a}$ as given by (1) is indeed possible. However, the Jacobian will not be needed in the calculations of this paper. Only the defining equation of the dreibein will be used.

\section{Nonperturbative Monopole Configurations and Conformal Mode of Metric}

It is expected that topological degrees of freedom like magnetic monopoles are responsible for nonperturbative properties such as confinement [23]. In the Georgi-Glashow model, confinement is due to the 't Hooft-Polyakov monopole solution [24]. In contrast to this model, there is no scalar (Higgs) field in the SO(3) Yang-Mills theory in three dimensions. But the gauge field part of the 't Hooft-Polyakov monopole with appropriate boundary conditions (see below) constitutes a finite-action field configuration and contributes to the functional integral. Moreover, it has strong qualitative effects on the Wilson loop.

We now summarize the idea of "centres" of topological field configurations as developed and used in our earlier works $[5,10,25]$. (This framework is in the spirit of, but different from, the Abelian projection procedure of 't Hooft [26].) The topological properties of the 't Hooft-Polyakov monopole can be characterised using only the gauge field and in the interior (in contrast to the usual characterisation by the triplet scalar field at infinity). This is therefore useful in pure gauge theory. This characterisation is done using the eigenvector fields of the gauge invariant quantity $S_{i j} \equiv B_{i}^{a} B_{j}^{a}$, where $B_{i}^{a} \equiv$ $(1 / 2) \epsilon_{i j k} F_{j k}^{a}$ is the non-Abelian magnetic field.

For the 't Hooft-Polyakov monopole, $S_{i j}=\alpha(r) \delta_{i j}+$ $\beta(r) x_{i} x_{j}$, where $\alpha$ and $\beta$ are functions of the distance $r$ from the origin. At $r=0, S_{i j} \sim \delta_{i j}$ and so any direction is an eigenvector. Thus the eigenvector $x_{i}$ with unit winding number is singular at the origin. In general, the points at which the eigenvalues of $S_{i j}$ become triply degenerate or, equivalently, the eigenvector fields of $S_{i j}$ become singular, are called the "centres" of the monopoles and other topological objects in Yang-Mills theory.

Let us then consider the gauge field part of the 't HooftPolyakov monopole configuration

$$
A_{i}^{a}(x)=\epsilon_{a i j} x_{j} \frac{1-K(r)}{r^{2}} .
$$

Here $K(r)=1+O\left(r^{2}\right)$ for $r \rightarrow 0$ and $K(r) \rightarrow 0$ for $r \rightarrow \infty$. (These conditions ensure the finiteness of the action.) Using

$$
e_{i}^{a}(x)=\delta_{i}^{a} e^{-g(r)}
$$

in (24), we find that the resulting gauge potential corresponds to $(40)$ with

$$
\frac{d g}{d r}=\frac{1-K(r)}{r} .
$$

The configurations (40) and (41) possess spherical symmetry. We next consider a generalization of (41) with $\phi(x)$, an arbitrary scalar function of $x_{1}, x_{2}$, and $x_{3}$, in the place of $g(r)$ :

$$
e_{i}^{a}(x)=\delta_{i}^{a} e^{-\phi(x)} .
$$

This corresponds to

$$
g_{i j}=\delta_{i j} e^{-2 \phi},
$$

a conformally flat metric, and we call $\phi$ the conformal mode. Using (43) in (24) now leads to

$$
A_{i}^{a}(x)=\epsilon_{a i j} \partial_{j} \phi
$$


This gives

$$
B_{i}^{a}=\partial_{i} \partial_{a} \phi-\delta_{i a} \partial^{2} \phi+\partial_{i} \phi \partial_{a} \phi
$$

Now consider a point at which the following hold:

(1) $\partial_{i} \phi=0$.

(2) $\partial_{i} \partial_{a} \phi=0$ for $i \neq a$.

(3) $\partial^{2} \phi / \partial x_{1}^{2}=\partial^{2} \phi / \partial x_{2}^{2}=\partial^{2} \phi / \partial x_{3}^{2}$.

Note that the first condition says that we have a critical point and the next two conditions say that the Hessian matrix is diagonal and its three diagonal elements (eigenvalues) are equal. These are sufficient conditions for $\phi$ to be an extremum at the point. ( $\phi$ attains a maximum or a minimum depending on whether the eigenvalues are all positive or all negative.)

Now since conditions 2 and 3 are equivalent to $\partial_{i} \partial_{a} \phi=$ $(1 / 3) \delta_{i a} \partial^{2} \phi$, it follows from (46) that, at the point under consideration, $B_{i}^{a} \sim \delta_{i a}$ and so $B_{i}^{a} B_{j}^{a} \sim \delta_{i j}$. So the matrix $S_{i j}$ is triply degenerate at the point, which, as explained at the beginning of this section, is the criterion for locating the "centre" of a topological configuration in a gauge-invariant description. Thus at the topological centre, the field $\phi$ is an extremum, satisfying the three conditions given above.

For the special, spherical symmetric case given by (40) and (41), using $K(r)=1+O\left(r^{2}\right)$ for $r \rightarrow 0$, it can be checked that the function $g(r)$ indeed satisfies the above conditions on $\phi(x)$ at the monopole centre $r=0$. On the other hand, in the more general form given by (43), the conditions on $\phi\left(x_{1}, x_{2}, x_{3}\right)$ stated above show that $\phi$ needs to be symmetric only up to the second-order terms in the Taylor expansion about the centre. Thus, taking the topological centre to be at $r=0$, the forms $A+B r^{2}+C x_{1}^{3}+\cdots$ and $A+B r^{2}+D x_{1}^{2} x_{2}+\cdots$ (where $A, B, C$, and $D$ are constants) are two examples of possible Taylor expansion for the function $\phi$ satisfying the conditions.

It is interesting to apply our formalism to the $\mathrm{Wu}$-Yang monopole [27]. This is a solution to the Yang-Mills field equations. However, it is a point-like magnetic monopole. The gauge potential for it is obtained on putting $K(r)=0$ in (40). Then (42) gives $g(r)=\ln r$ (up to an additive constant, which sets the scale). So the conformal mode goes to negative infinity as $r$ goes to zero. Such a singularity, instead of a smooth minimum, in the conformal mode is just what is expected since the $\mathrm{Wu}$-Yang monopole is point-like. The metric in this case is $g_{i j}=\delta_{i j} / r^{2}$ (up to a multiplicative constant).

\section{Ansatz for Gauge Field}

We now consider the polar decomposition of the most general $3 \times 3$ matrix $e_{i}^{a}$ into an orthogonal matrix $\mathscr{R}$ and a symmetric matrix $\mathscr{E}$ :

$$
e_{i}^{a}(x)=\mathscr{R}_{j}^{a}(x) \mathscr{E}_{j i}(x) \quad \forall x .
$$

Under a gauge transformation,

$$
\mathscr{R}_{j}^{a}(x) \longrightarrow O^{a b}(x) \mathscr{R}_{j}^{b}(x),
$$

while the symmetric matrix $\mathscr{E}_{j i}$ is gauge-invariant and is the symmetric square-root of the metric $g_{i j}$. The decomposition (47) corresponds to

$$
A_{l}^{d}(x)=\mathscr{R}_{m}^{d}(x) a_{m l}(x)+\omega_{l}^{d}(x)
$$

where

$$
a_{m l}(x)=\frac{1}{\operatorname{det} \mathscr{E}} \epsilon_{i j k}\left(\mathscr{E}_{i m} \mathscr{E}_{l p}-\frac{1}{2} \mathscr{E}_{i p} \mathscr{E}_{l m}\right) \partial_{j} \mathscr{E}_{k p}
$$

and $\omega_{l}^{d}$ is formally a pure gauge:

$$
\omega_{l}^{d}(x)=-\frac{1}{2} \epsilon^{b c d} \mathscr{R}_{m}^{b}(x) \partial_{l} \mathscr{R}_{m}^{c}(x)
$$

(To obtain (49), one puts (47) in (24). The terms containing derivative of $\mathscr{E}$ immediately give the first term on the RHS of (49). In the terms containing derivative of $\mathscr{R}$, we put $\partial_{j} \mathscr{R}_{p}^{a}=-\epsilon^{a b c} \omega_{j}^{b} \mathscr{R}_{p}^{c}$, which is the same as (51). Then using $\epsilon^{a b c} \mathscr{R}_{n}^{a} \mathscr{R}_{p}^{c}=\epsilon_{n q p} \mathscr{R}_{q}^{b}$ (since $\left.\operatorname{det} R=1\right)$ and $\epsilon_{i j k} \mathscr{E}_{i n} \mathscr{E}_{k p}=$ $\epsilon_{n r p}(\operatorname{det} \mathscr{E})\left(\mathscr{E}^{-1}\right)_{j r}$, we get the $\omega_{l}^{d}$ term in (49).)

Now the symmetric matrix $\mathscr{E}$ can be further decomposed into a spin-two traceless part and a scalar trace part:

$$
\mathscr{E}_{i j}=\widetilde{\mathscr{E}}_{i j}+\delta_{i j} e^{-\phi}
$$

(Here $\widetilde{\mathscr{E}}_{i j}=\mathscr{E}_{i j}-(1 / 3) \delta_{i j} \Sigma_{k} \mathscr{E}_{k k}$ and we define $(1 / 3) \Sigma_{k} \mathscr{E}_{k k}$ to be $e^{-\phi}$.) Since $\delta_{i j} e^{-\phi}$ corresponds to the metric (44), we propose that the topological configurations are contained in this part, with $\phi$ satisfying the three conditions given after (46) at isolated points. At such points, $\omega_{l}^{d}$ is not strictly a pure gauge. For example, the non-Abelian magnetic field corresponding to $\omega_{l}^{d}$ has a Dirac string contribution when $\mathscr{R}$ is the singular gauge in which the configuration (40) becomes a Dirac monopole [10].

Thus (49), (50), (51), and (52) comprise our ansatz for the gauge potential, which contains the topological aspects in a natural way. The three gauge degrees of freedom reside in $\mathscr{R}$ and the six gauge-invariant degrees of freedom reside in $\mathscr{E}$. The conformal mode contained in $\mathscr{E}$ captures the topological configurations.

\section{Discussion}

In this work, we have used the dreibein and the metric as basic variables for the SO(3) Yang-Mills theory in three Euclidean dimensions. The usefulness of this lies in the fact that the metric is gauge invariant.

By showing that a generic $A_{i}^{a}$ corresponds to a unique torsion-free $e_{i}^{a}$ on fixing the boundary condition, we have provided justification for the change of variables from $A_{i}^{a}$ to $e_{i}^{a}$.

We have applied this formalism to nonperturbative aspects of the theory. The conformal mode of the metric is found to contain the topological aspects of the gauge potential. We have demonstrated that topological centres are located at certain points at which this conformal mode, a scalar field, is an extremum. This criterion is thus an 
alternative to that developed by us in $[5,10]$ (also applied by us in [25]), namely, that the points of triple degeneracy of $B_{i}^{a} B_{j}^{a}$ are the centres of topological configurations. Both of these criteria are gauge invariant. The Yang-Mills theory expressed in terms of this conformal mode would be a theory of a scalar field, and lattice simulation of this scalar theory (as opposed to lattice gauge theory) with monopoles located at extrema of the scalar field would serve as a model for confinement.

We have also proposed an ansatz for the gauge potential, which incorporates the topological aspects contained in the conformal mode after separating the gauge variant and the gauge invariant degrees of freedom.

When our formalism is applied to the Hamiltonian formulation of $3+1$-dimensional Yang-Mills theory, the canonical variables are the metric and its conjugate variable. The physical wave functionals are simply functionals of the metric. However, due to the change of variables, the inner product of the wave functionals will involve the Jacobian of the transformation. For $2+1$-dimensional $S U(N)$ gauge theory, Nair et al. [28-31] proposed an $N \times N$ complex matrix as a prepotential and, as a consequence of the change of variables, obtained an inner product for physical states which involves the Wess-Zumino-Witten action. They arrived at a trial wave functional which exhibits confinement and gives string tension in striking agreement with lattice gauge theory simulations. Viewing our proposal of using the dreibein as prepotential as a generalization to $3+1$-dimensions, it will therefore be interesting to calculate the Jacobian of the transformation in our case.

\section{Conflicts of Interest}

The authors declare that there are no conflicts of interest regarding the publication of this paper.

\section{Acknowledgments}

Indrajit Mitra thanks UGC (DRS) for support.

\section{References}

[1] P. E. Haagensen and K. Johnson, "Yang-Mills fields and Riemannian geometry," Nuclear Physics B, vol. 439, no. 3, pp. 597-616, 1995.

[2] D. Z. Freedman, P. E. Haagensen, K. Johnson, and J. I. Latorre, https://arxiv.org/abs/hep-th/9309045.

[3] M. Bauer, D. Z. Freedman, and P. E. Haagensen, "Spatial geometry of the electric field representation of non-abelian gauge theories," Nuclear Physics B, vol. 428, no. 1-2, pp. 147-168, 1994.

[4] F. A. Lunev, "Three dimensional yang-mills theory in gauge invariant variables," Physics Letters B, vol. 295, no. 1-2, pp. 99103, 1992.

[5] R. Anishetty, P. Majumdar, and H. S. Sharatchandra, "Dual gluons and monopoles in 2+1 dimensional Yang-Mills theory," Physics Letters B, vol. 478, pp. 373-378, 2000.

[6] P. Majumdar and H. S. Sharatchandra, "(3+1)-dimensional Yang-Mills theory as a local theory of evolution of metrics on 3-manifolds," Physics Letters B, vol. 491, no. 1-2, pp. 199-202, 2000 .
[7] D. Diakonov and V. Petrov, "Gauge-invariant formulation of the $d=3$ Yang-Mills theory," Physical Review Letters, vol. 493, no. 1-2, pp. 169-174, 2000.

[8] S. Nojiri and S. D. Odintsov, "Entropy bounds and CardyVerlinde formula in Yang-Mills theory," Physics Letters B, vol. 540, no. 3-4, pp. 167-172, 2002.

[9] P. E. Haagensen, K. Johnson, and C. S. Lam, "Gauge invariant geometric variables for Yang-Mills theory," Nuclear Physics B, vol. 477, no. 1, pp. 273-292, 1996.

[10] E. Harikumar, I. Mitra, and H. S. Sharatchandra, "Topological field patterns of the Yang-Mills theory," Physics Letters B, vol. 557, no. 3-4, pp. 297-302, 2003.

[11] Y. M. Cho, "Restricted gauge theory," Physical Review D, vol. 21, p. 1080, 1980.

[12] L. Faddeev and A. J. Niemi, "Partially Dual Variables in SU(2) Yang-Mills Theory," Physical Review Letters, vol. 82, p. 1624, 1999.

[13] L. D. Faddeev and A. J. Niemi, "Decomposing the Yang-Mills field," Physics Letters B, vol. 464, no. 1-2, pp. 90-93, 1999.

[14] L. D. Faddeev and A. J. Niemi, "Aspects of electric and magnetic variables in SU(2) Yang-Mills theory," Physics Letters B, vol. 525, no. 1-2, pp. 195-200, 2002.

[15] A. J. Niemi, “Three-dimensional gravity from SU(2) Yang-Mills theory in two dimensions," Physical Review D, vol. 70, 2004.

[16] L. D. Faddeev and A. J. Niemi, "Spin-charge separation, conformal covariance and the $S U(2)$ Yang-Mills theory," Nuclear Physics B, vol. 776, no. 1-2, pp. 38-65, 2007.

[17] E. Witten, " $2+1$ dimensional gravity as an exactly soluble system," Nuclear Physics B, vol. 311, no. 1, pp. 46-78, 1988.

[18] A. Ashtekar, "New variables for classical and quantum gravity," Physical Review Letters, vol. 57, no. 18, pp. 2244-2247, 1986.

[19] K. Krasnov, "A gauge-theoretic approach to gravity," Proceedings of the Royal Society A: Mathematical, Phisical and Engineering Sciences, 2012.

[20] Z.-Q. Guo, "Reformulations of Yang-Mills theories with spacetime tensor fields," Annals of Physics, vol. 364, pp. 283-316, 2016.

[21] G. 't Hooft, "A chiral alternative to the vierbein field in general relativity," Nuclear Physics B, vol. 357, no. 1, pp. 211-221, 1991.

[22] P. Majumdar and H. S. Sharatchandra, "Gauge field copies," Physical Review D, vol. 63, p. 067701, 2001.

[23] S. Mandelstam, "II. Vortices and quark confinement in nonAbelian gauge theories," Physics Reports, vol. 23, no. 3, pp. 245249, 1976.

[24] A. M. Polyakov, "Quark confinement and topology of gauge theories," Nuclear Physics B, vol. 120, no. 3, pp. 429-458, 1977.

[25] E. Harikumar, I. Mitra, and H. S. Sharatchandra, "Half-monopoles and half-vortices in the Yang-Mills theory," Physics Letters $B$, vol. 557, no. 3-4, pp. 303-308, 2003.

[26] G. 't Hooft, “Topology of the gauge condition and new confinement phases in non-abelian gauge theories," Nuclear Physics B, vol. 190, no. 3, pp. 455-478, 1981.

[27] T. T. Wu and C. N. Yang, Properties of Matter Under Unusual Conditions, vol. 23, Interscience, New York, NY, USA, 1968.

[28] D. Karabali and V. P. Nair, "A gauge-invariant Hamiltonian analysis for non-Abelian gauge theoreiesin $(2+1)$ dimensions," Nuclear Physics B, vol. 464, 135, 1996.

[29] D. Karabali and V. P. Nair, "On the origin of the mass gap for non-Abelian gauge theories in $(2+1)$ dimensions," Physics Letters $B$, vol. 379, no. 1-4, pp. 141-147, 1996. 
[30] D. Karabali, C. Kim, and V. P. Nair, "Planar yang-mills theory: hamiltonian, regulators and mass gap," Nuclear Physics B, vol. 524, no. 3, pp. 661-694, 1998.

[31] V. P. Nair, The Hamiltonian Approach to Yang-Mills (2+1): An Update and Corrections to String Tension, Proceedings of Science, 2009. 

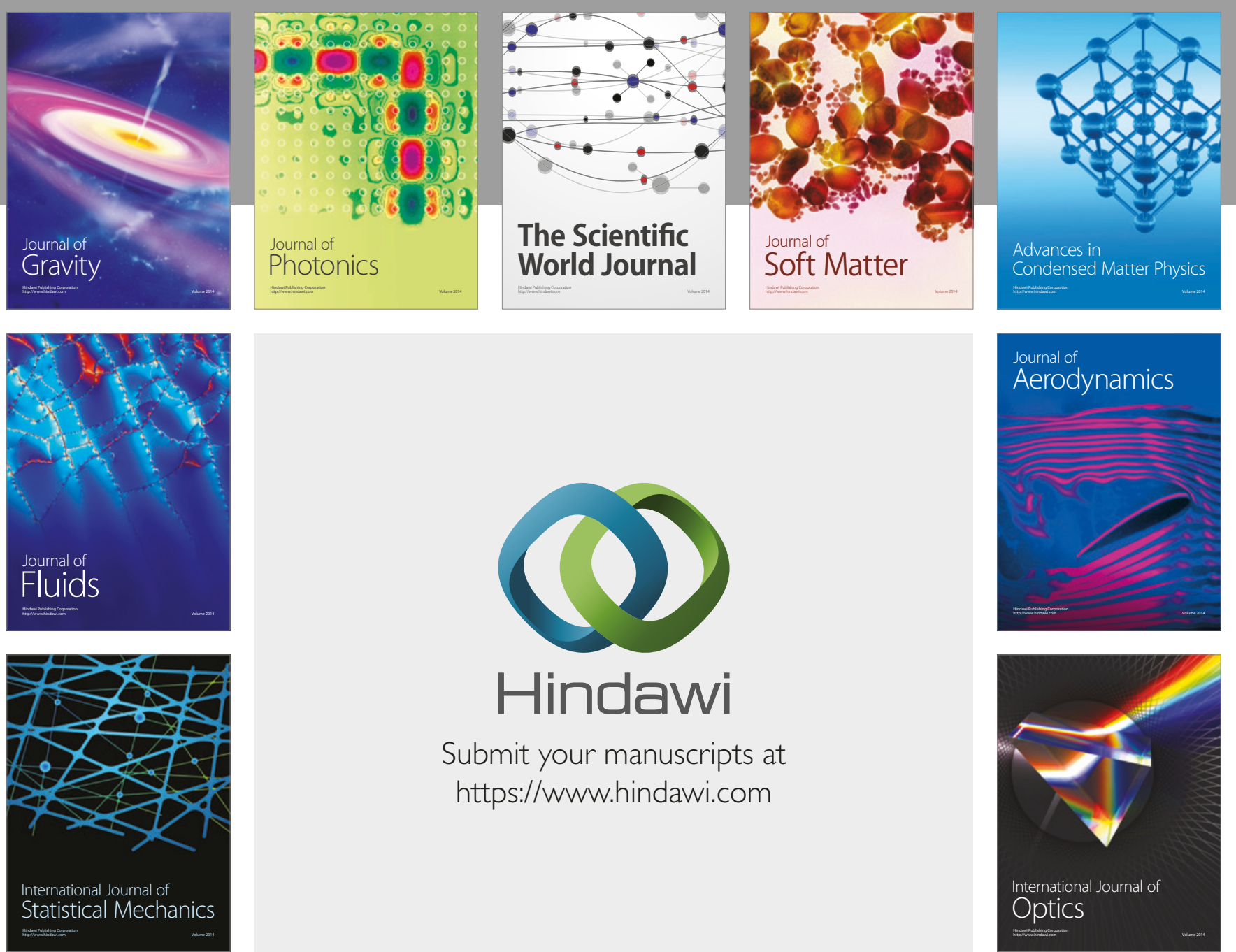

Submit your manuscripts at

https://www.hindawi.com
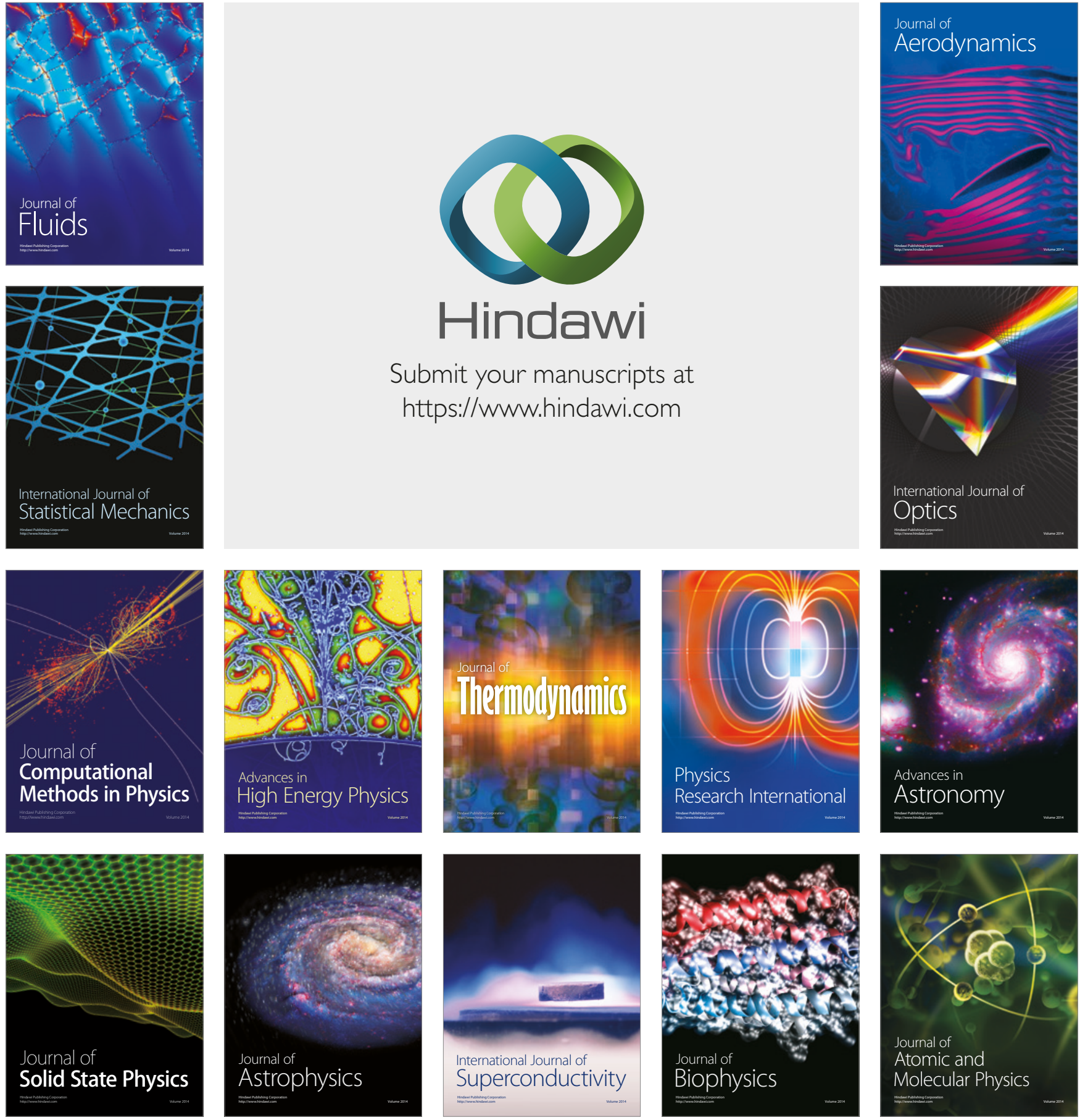